Le Centre de Recherches en Histoire

Internationale et Atlantique des Universités

de Nantes et de La Rochelle, en collaboration avec les institutions patrimoniales de

Rochefort, ouvre une série de journées d'etudes annuelles autour de l'histoire du gouvernement des hommes en mer.

Ce projet de recherche pluridisciplinaire

Histoire, Littérature, Philosophie - observe les gens de

mer par leur organisation politique. Le contrôle des équipages embarqués dans des espaces confinés exigeait en effet qu'un capitaine, seul maître à bord, fasse usage de rigueur dans sa conduite des hommes. Un pouvoir exceptionnel en résulte, pouvoir qui confere à la structure de commandement des navires une autorité renforcée et limitée par le temps d'une traversée.

Cette expérience singulière, trempée dans les flots océaniques, inspire littératures et réflexions politiques. Si elle est à l'origine d'un imaginaire de la mer, elle nourrit également nos libertés publiques, éprouvées par les contraintes de la houle. Des mutineries, aux châtiments à bord, en passant par les pratiques cultuelles et le rôle des écritures embarquées, il est un récit politique de la mer à écrire. Entre utopie et tyrannie, le "gouvernement des hommes en mer » en tentera l'esquisse.
Ancienne École de Médecine

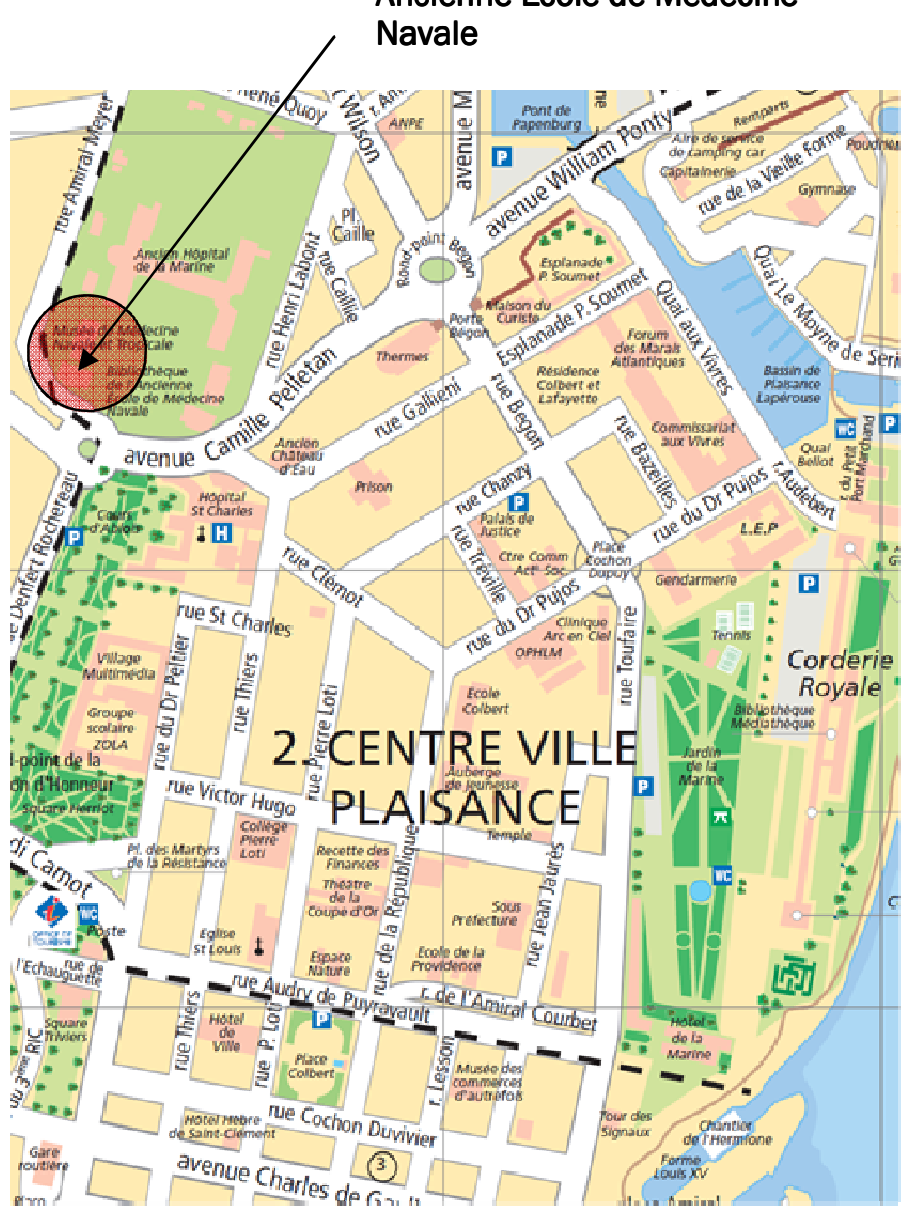

IRE INTERNATIONALE ET ATLANTRQUE

UNIVERSITE DE LA ROCHELLE

Courriel : marielle.juhe!@univ-lr.fr

ÉCOLE dE MÉdecine NaVAle (MusÉe NATIONAL de LA MARINE)

25, rue de l'Amiral Meyer, 17300 Rochefort.

Tel : 0546992601

Courriel : d.roland@musee-marine.fr

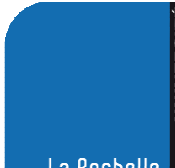

Journée d'études:

"Le gouvernement des

hommes en mer, I

\section{Fureur et cruauté des capitaines en mer}

Organisation : Pierre Prétou et Denis Roland

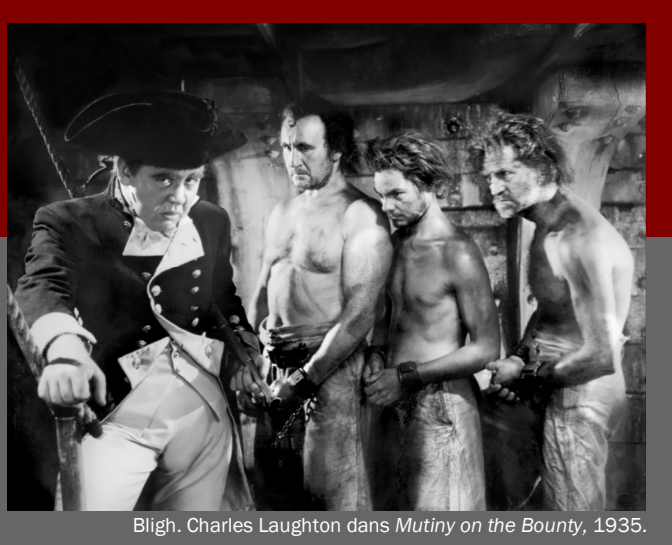

Rochefort, École de médecine navale, 1 er fufllet 2009 9h30-12h00 et 14h30-17h00 de la Marine 


\section{Fureur et cruauté des capitaines en mer}

Vent debout, la sévérité des capitaines envers leur équipage alimente un motif classique des représentations du commandement maritime. De l'Antiquité à nos jours, les justifications de cette rigueur paraitront diverses, qu'elles soient une nécessité militaire impérieuse, une contrainte liée au danger, ou une conséquence de la vie collective embarquée dans des espaces confinés.

Surgis de notre imaginaire ou histoire, les figures des capitaines Bligh, Achab ou Nemo scellent cette idée selon

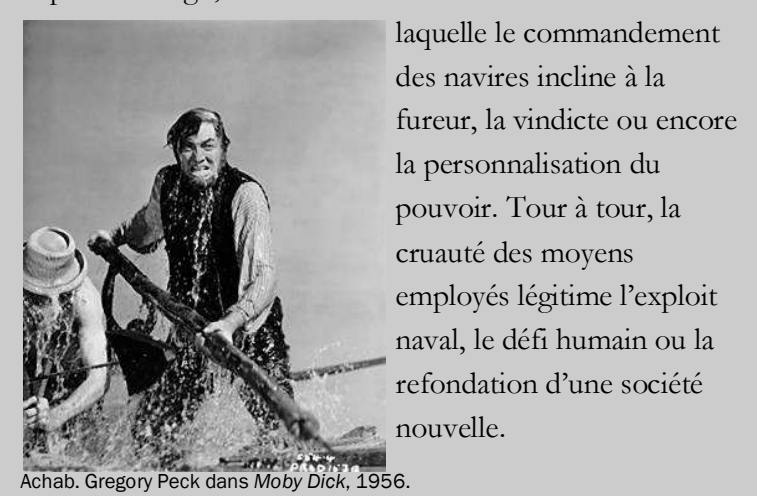

Ces histoires ne sont pas de simples récits isolés : elles éprouvent notre conscience politique aux périphéries

hostiles du monde habité et trouvent ainsi dans l'océan un des fondements peu étudié de la pensée politique.

Les figures de la fureur ou de la cruauté des capitaines en mer sont donc une des portes d'entrée permettant l'appréhension symbolique de l'histoire du commandement naval. Parce qu'elles cristallisent dans l'homme un pouvoir exceptionnel, son exercice féroce, anxiogène ou tyrannique se mesure aussi à l'aune de ses finalités contradictoires: une libération des hommes ou un affranchissement de servitude terrestre.
Accueil des participants et présentation

Denis RoLAND, Musée national de la Marine

Introduction générale : Satan maitre à bord et la damnation maritime dan les manuscrits français de la fin du Moyen Age.

Pierre PrÉTOU, MCF, Histoire du Moyen Âge

Matinée

Présidence Michel BocHACA, PR, Histoire médiévale.

$$
10 h 00-11 \text { b00 : la vindicte en mer }
$$

Malheur aux vaincus, malbeur pour les vainqueurs! : à propos de la cruauté légendaire des marins étrusques.

Laurent HUGOT, MCF, Histoire ancienne

Étude comparée d'une figure de la cruauté : les capitaines Achab dans Moby Dick d'Herman Melville et Bligh dans Les Révoltés de la Bounty de Jules Verne.

Charles BRION, MCF, Littérature comparée

$$
11 \text { h00 — 12h00 : l'angoisse des passagers }
$$

Le capitaine du Nanshan, son second et les «coolies» chinois embarqués dans Typhoon de Joseph Conrad.

Yvan DANIEL, MCF HDR, Littérature comparée

L'attente à bord des navires d'immigrants an XIX $X^{e}$ siècle : bypothèses de travail.

Laurent VIDAL, PR, Histoire contemporaine

$$
\text { Après-midi }
$$

Présidence Didier POTON, PR, Histoire moderne.

$$
14 h 30-15 h 30: \text { les pouvoirs néfastes }
$$

Être le fils d'un capitaine cruel : trace et effacement d'un terrible béritage $d u$ Voyage en Amérique aux Mémoires d'Outre Tombe de Chateaubriand.

Serge LINKÉS, MCF, Littérature française

La tyrannie du charbon et de la machine lors des débuts de la marine à vapeur au XIXe siècle : la Royale lors de l'expédition an Mexique. Romain DeLMON, PRAG, Histoire contemporaine
Autorité et confiance a bord : capitaines et seconds chez Conrad. Laurence CORNU, MCF HDR, Philosophie

Figures de traumatisme en mer: les chirurgiens embarqués face aux équipages, fin XVIII' — début XIX'e siècle.

Grégory BÉRIET, Dr, Histoire contemporaine

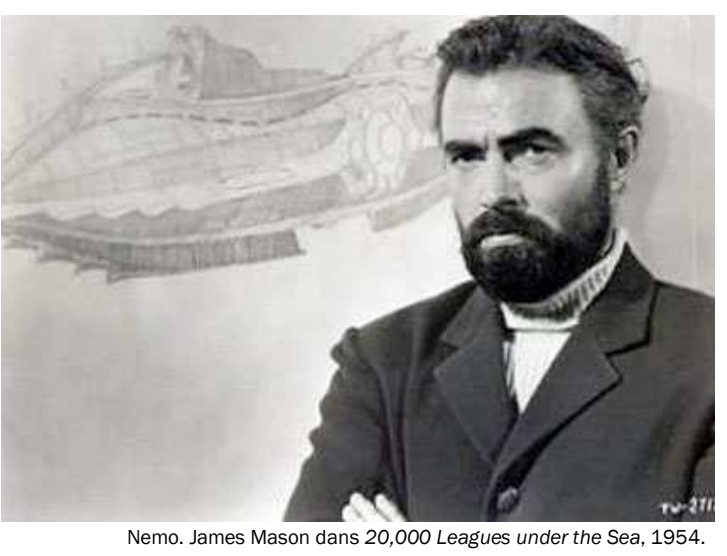

Fin d'après-midi

Présidence Charles ILlOUZ, PR, Anthropologie.

$16 h 30-17$ h00 : le mal sur mer, débats et conclusions de la journée

centrederecherches en

Histoire Internationale

e $t$ A $t$ I a n t i q u e

Centre de Recherches en Histoire Internationale et Atlantique : CRHIA EA 1163, Nantes - La Rochelle. Université de La Rochelle

Arts et Sciences Humaines

17042 La Rochelle Cedex 1

Contact et renseignements : Marielle Juhel

0546458559

marielle.juhel@univ-Ir.fr

Organisation : Pierre Prétou et Denis Roland pierre.pretou@univ-Ir.fr; d.roland@musee-marine.fr 